\title{
Trends in Cervical Cancer Among Delivery-Related Discharges and its Impact on Maternal-Infant Birth Outcomes (United States, 1998-2009)
}

\author{
Mulubrhan F. Mogos ${ }^{*}, 1$, Jason L. Salemi ${ }^{2}$, Dawood H. Sultan ${ }^{3}$, Melissa M. Shelton ${ }^{4}$ and \\ Hamisu M. Salihu ${ }^{2,5}$
}

\author{
${ }^{I}$ Department of Women Children and Family Health Science, College of Nursing, University of Illinois, 845 S Damen \\ Ave, Chicago, IL 60612, USA \\ ${ }^{2}$ Maternal and Child Health Comparative Effectiveness Research Group, Department of Epidemiology and Biostatistics, \\ College of Public Health, University of South Florida, 13201 Bruce B. Downs Blvd., MDC 56, Tampa, FL 33612, USA \\ ${ }^{3}$ College of Public Health, University of South Florida, Department of Health Policy and Management; 13201 Bruce B. \\ Downs Blvd., Tampa, FL 33612, USA \\ ${ }^{4}$ College of Nursing, University of South Florida, 12901 Bruce B. Downs Blvd., MDC Box 22, Tampa, FL 33612, USA \\ ${ }^{5}$ Division of Maternal-Fetal Medicine, Department of Obstetrics and Gynecology, Morsani College of Medicine, \\ University of South Florida, 12901 Bruce B. Downs Blvd., Tampa, FL 33612, USA
}

\begin{abstract}
Objectives: To estimate the national prevalence of cervical cancer (CCA) in women discharged from hospital after delivery, and to examine its associations with birth outcomes.

Methods: We did a retrospective cross-sectional analysis of maternal hospital discharges in the United States (1998-2009). We used the Nationwide Inpatient Sample (NIS) database to identify hospital stays for women who gave birth. We determined length of hospital stay, in-hospital mortality, and used ICD-9-CM codes to identify CCA and all outcomes of interest. Multivariable logistic regression modeling was used to calculate adjusted odds ratios (AOR) and $95 \%$ confidence intervals (CI) for the associations between CCA and feto-maternal outcome.

Results: In the 12-year period from 1998 to 2009, there were 8,387 delivery hospitalizations with a CCA diagnosis, a prevalence rate of 1.8 per 100,000 $(95 \% \mathrm{CI}=1.6,1.9)$. After adjusting for potential confounders, CCA was associated with increased odds of maternal morbidities including: anemia (AOR, 1.78, 95\% CI, 1.54-2.06), anxiety (AOR, 1.95, 95\% CI, 1.11-3.42), cesarean delivery (AOR, 1.67, 95\% CI, 1.46-1.90), and prolonged hospital stay (AOR, 1.51, 95\% CI, 1.301.76), and preterm birth (AOR, 1.69, 95\% CI, 1.46-1.97).

Conclusion: There is a recent increase in the prevalence of CCA during pregnancy. CCA is associated with severe fetomaternal morbidities. Interventions that promote safer sexual practice and regular screening for CCA should be promoted widely among women of reproductive age to effectively reduce the prevalence of CCA during pregnancy and its impact on the health of mother and baby.
\end{abstract}

Keywords: Birth outcomes, cervical cancer, delivery, fetus, maternal, trend.

\section{INTRODUCTION}

In recent years, the average age at which a woman delivers her first child has been increasing [1]. Considering this delay in childbearing to older ages, and the association between later age at first delivery and increased risk of several types of cancer, the expectation would be an increase in the number of women with a diagnosis of cancer during pregnancy [2]. Cervical cancer is one of the leading types of reproductive cancer among pregnant mothers [3]. Recently, a study of nine Surveillance, Epidemiology, and End Results (SEER) registries investigated trends of cervical cancer in the

*Address correspondence to this author at the Department of Women Children and Family Health Science, College of Nursing, University of Illinois, $845 \mathrm{~S}$ Damen Ave, Chicago, IL 60612, USA; E-mail: talk2mulie@gmail.com
United States (US) and reported an overall 54\% reduction in the incidence of invasive cervical cancer over a 35 -year period, from 13.07 per $100,000(1973-1975)$ to 6.0 per 100,000 (20062007) [4]. However, despite the comprehensive nature of their analyses, the authors did not look specifically at the rates and temporal trends of cervical cancer during pregnancy. Pregnancy provides a valuable opportunity to screen and diagnose women for cervical cancer as they are receiving routine antenatal care.

A recent study in Denmark reported that incidence of pregnancy-associated cancer, including cervical cancer, has been rising over the past 30 years [5]. These increases have been attributed largely to the aforementioned tendency for women to give birth at later ages, but also to increased detection rates due to an increased frequency of encounters with the healthcare system during pregnancy $[4,6]$. An analysis of delivery hospitalizations in countries like the US, 
where a high preponderance of deliveries occur in hospitals and other healthcare facilities [7] should provide improved estimates of the prevalence of pregnancy-associated cervical cancer. The National Inpatient Sample (NIS) series of databases, made available through the Healthcare Cost and Utilization Project (HCUP), makes it possible to investigate national rates and temporal trends of cervical cancer among pregnancy women. Furthermore, the availability of patient and hospital-level socio-demographic and geographic characteristics such as age, race, and urbanization in the NIS can facilitate analyses that identify particular subsets of pregnant women that may be at increased risk for cervical cancer, and can serve as a proxy for assessing the effectiveness of cervical cancer prevention programs in different regions of the US.

Having up-to-date, national estimates of the frequency and rate of pregnancy-related cervical cancer and understanding its association with maternal-fetal outcomes is critical to public health planning, resource allocation, and the provision of reliable data to decision-makers. This study fulfills that role as it aims to: 1) estimate the rates and 12year trends of cervical cancer among delivery-related hospitalizations in the US, overall and by patient and hospital characteristics; 2) investigate whether a cervical cancer diagnosis during pregnancy is associated with adverse maternal-fetal outcomes; and 3) estimate the impact of cervical cancer during pregnancy on the direct cost of medical care.

\section{METHODS}

\section{Study Design and Data Source}

We conducted a cross-sectional analysis of hospital discharges in which a singleton delivery occurred, using 1998-2009 annual data from the NIS, which is the largest allpayer, publicly available inpatient database in the US [8]. The NIS dataset is created using a sampling technique that stratifies all non-federal community hospitals from states into groups based on five major hospital characteristics: rural/urban location, number of beds, geographic region, teaching status, and ownership. A 20\% sample of hospitals is randomly drawn from each stratum and all inpatient discharge records from selected hospitals are included. The NIS database comprises hospital stratum identifiers and discharge-level sampling weights whose purpose is to facilitate the generation of national prevalence estimates.

\section{Identifying Maternal Cases and Clinical Conditions}

Delivery-related hospitalizations were identified using a two-step process. First, we used an HCUP-created variable, NEOMAT, designed to classify hospitalizations as maternal and/or neonatal [9]. Then we used the presence of a "V27.0" or "V27.1" International Classification of Diseases, Ninth Edition, Clinical Modification (ICD-9-CM) code to select women who had a singleton delivery during their hospitalization, either live- or stillborn. Each hospital discharge record contains ICD-9-CM codes for a patient's principal diagnosis and up to 14 secondary diagnoses. Beginning in 2009, the NIS included up to 24 secondary diagnosis fields [10]. Once restricted to delivery hospitalizations, we identified women with a cervical cancer diagnosis using the presence of one or more of the following ICD-9-CM codes: 180.0, 180.1, 180.8, 180.9, 233.1, and 795.06. Maternal co-morbidities and maternal-fetal outcomes, including early onset delivery and poor fetal growth, were also identified using ICD-9-CM codes. A detailed list of the specific ICD-9-CM diagnosis and procedure codes used in this study are presented in Appendix $A$

We used the maternal length of stay (LOS) during the delivery hospitalization as an indicator of healthcare utilization and as a proxy for the severity of complications during delivery. We defined a prolonged hospitalization as a LOS as one that met or exceeded the $95^{\text {th }}$ percentile based on the distribution among all delivery hospitalizations ( $\geq 4$ days in our sample).

\section{Demographics and Covariates}

To explore differences in baseline characteristics, we compared the distribution of selected socio-demographic, behavioral, and hospital characteristics of those with and without a cervical cancer diagnosis. We grouped maternal age in years into five clinically meaningful categories: $<20$, $20-24,25-29,30-34$, and $\geq 35$. Race was determined first by self-reported ethnicity (Hispanic or non-Hispanic), with the non-Hispanic $(\mathrm{NH})$ group further subdivided by race (white, black, or other). Household income was estimated by HCUP using the mother's zip code of residence at delivery, and subsequently ranked into quartiles [8]. The primary payer for each hospitalization was classified into government (Medicare/Medicaid), private (commercial carriers and private HMOs and PPOs), or other (including self-pay and no charge). Hospital characteristics assessed included teaching status (teaching $v s$ non-teaching), location (urban $v s$ rural), and US geographic region (Northeast, Midwest, South, or West).

\section{Cost Estimation}

To examine the economic impact of pregnancy-related cervical cancer, hospital charges that are reported for each hospitalization were converted to a more reliable estimate of cost. Hospital charges reflect what a hospital bills for services, whereas the desired measure should represent an estimate of actual resource consumption. Markup for services from actual cost to what is charged varies over time, across hospitals, and even among different departments within the same hospital $[11,12]$. To estimate the actual cost of direct medical care, we first multiplied the total charge of the hospitalization by a time- and hospital-specific cost-tocharge ratio (CCR) provided by HCUP [13]. Then, we multiplied reported charges by an "adjustment factor" (AF) from HCUP. The AF adjusts for bias that results from interdepartmental variations in markup within each hospital [14]. For example, within the same hospital, the markup for a surgical procedure may be considerably higher than for a routine bed service; the AF attempts to adjust for those differences. The formula below summarizes the conversion of hospital charges into a refined cost estimate: 


\section{Total Cost $=$ Total Charges $*$ Hospital-Specific CCR $* A F$}

\section{Statistical Analysis}

Descriptive statistics were used to estimate the national rate of cervical cancer among women delivering singleton offspring. All estimates were weighted to account for the complex sampling design of the NIS. We assessed the distribution of socio-demographic, behavioral, hospital characteristics, and selected clinical and perinatal conditions by cervical cancer status. Temporal trends in rates of cervical cancer during the 12-year study period were assessed using joinpoint regression. Joinpoint regression provides the best fitting model with time points where the rate changes (increase or decrease) significantly [15]. Each distinct temporal trend identified by the joinpoint model is described using the annual percent change [APC, average annual change over the years during a particular trend] and the average annual percent change [AAPC, an overall APC that characterizes the entire study period, even if there were statistically significant changes in the trend] [15]. For temporal analyses we used the NIS-Trends files, supplied by HCUP to ensure consistency in the trend weights and in definitions of data elements over time [16].

Survey logistic regression modeling was used to estimate adjusted odds ratios (AOR) and 95\% confidence intervals (CI) for the association between pregnancy-related cervical cancer and each outcome of interest. For each outcome, we constructed a crude (unadjusted) model and two multivariable (adjusted) models. Covariates were identified through a review of the literature and empirical bivariate analyses. In the first multivariable model, we adjusted for maternal demographics (age and race/ethnicity, household income, multiple gestations), maternal behaviors (tobacco, alcohol, and drug use), clinically diagnosed obesity, primary payer, and rural/urban status. In the second multivariable model, in addition to covariates in the first multivariate model, we also controlled for clinical and pregnancy-related complications. These complications were captured using a composite binary variable that indicated the presence or absence these conditions (see Table 2 for list of variables included in the composite variables). The individual clinical and pregnancyrelated conditions used to create the clinical and pregnancyrelated composite variables were also identified using ICD-9$\mathrm{CM}$ codes. Conditions were selected based on their documented association with our outcomes of interest.

To estimate the impact of pregnancy-related cervical cancer on the cost of inpatient care at delivery, we compared the mean, per women, direct medical cost between discharges with and without a cervical cancer diagnosis. Economic analyses were restricted to 2001-2009 since reliable CCRs to convert charges to costs were not available prior to 2001. Statistical analyses were performed with SAS software, version 9.3 (SAS Institute, Inc., Cary, NC) and the Joinpoint Regression Program, version 4.0.1. [17]. This study was considered exempt from institutional review board approval because of the de-identified nature of the data.

\section{RESULTS}

In the 12-year period from 1998 to 2009, there were 8,387 delivery hospitalizations with a cervical cancer diagnosis, a prevalence rate of 1.8 per $100,000(95 \% \mathrm{CI}=1.6$, 1.9). Over 60,000 (128 per 100,000) discharge records also had a diagnosis of one or more sexually transmitted infections (STI). Among the 47,446,658 delivery hospitalizations, the maternal age distribution was as follows: teenagers $(10.9 \%), 20-34$ years $(75.1 \%)$, and advanced maternal age $(14.0 \%)$. Most reported their race as white-NH (39.9\%), followed by Hispanic (17.3\%), black-NH $(9.8 \%)$ and other-NH (7.6\%). Over $25 \%$ of hospitalizations were missing race/ethnicity, a known limitation of state reporting to the NIS. Private insurance (including HMO) was the primary payment source $(54.1 \%)$ followed by Medicaid/Medicare (39.5\%), and others including self-pay and missing primary payer information (6.4\%). The South accounted for $36.9 \%$ of delivery hospitalizations followed by West (24.8\%), Midwest (21.6\%) and Northeast (16.7\%). $87.5 \%$ of the discharges were from urban hospitals and $12.5 \%$ from rural hospitals.

We assessed whether certain demographic, behavioral, hospital, and clinical conditions were associated with an increased odds of cervical cancer using multivariable logistic regression. After adjusting for year of discharge and all variables listed in Table $\mathbf{1}$ (tobacco use, alcohol use, drug abuse, being from the south or west region, low household income, diagnosis of human immunodeficiency virus [HIV] or another STI), a number of characteristics were associated with pregnancy-related cervical cancer. Women who smoke during pregnancy were more likely to be diagnosed with cervical cancer when compared to women who didn't smoke $(\mathrm{AOR}=4.36 ; 95 \% \mathrm{CI}: 3.74,5.09)$. Other factors associated with increased risk of cervical cancer include: alcohol use $(\mathrm{AOR}=5.51 ; 95 \% \mathrm{CI}: 2.44,12.46)$; drug abuse $(\mathrm{AOR}=2.71$; 95\% CI: 1.93, 3.81); diagnosis of STD (AOR $=2.67,95 \% \mathrm{CI}$ : $1.67,6.09)$ and HIV (AOR=6.19; 95\% CI: 3.55, 10.79); presence of one or more maternal clinical conditions $(\mathrm{AOR}=1.56,95 \% \mathrm{CI}: 1.29,1.89)$ or pregnancy-related complications $(\mathrm{AOR}=1.19,95 \% \mathrm{CI}$ : 1.04, 1.37). Compared to women from the highest household income areas, those with progressively lower household income had a 1.54 to 2.30 times higher odds of pregnancy-related cervical cancer. Those from the Midwest and South regions had 39\% and $36 \%$ greater odds of cervical cancer, respectively, when compared to their counterparts from the West.

During the study period, the number of discharges with a cervical cancer diagnosis varied in frequency from a high of 831 in 1998 to a low of 534 in 2006 . Overall, we observed a non-significant $14.3 \%$ annual reduction in the rate from 1998 to 2001 followed by a statistically significant $4.1 \%(95 \% \mathrm{CI}$ : $0.1,8.2$ ) annual increase from 2001 to 2009 (Fig. 1). Subgroup analysis of temporal trends by region showed a statistically significant $6.3 \%$ annual reduction in the rate of pregnancyrelated cervical cancer in the Northeast region. However, in the South and West regions there were no statistically significant trends in cervical cancer rates. 
Table 1. Distribution of maternal socio-demographic, perinatal, behavioral, and hospital characteristics among delivery-related discharges, by cervical cancer (CCA) diagnosis status, HCUP-NIS, 1998-2009.

\begin{tabular}{|c|c|c|c|c|}
\hline Characteristic & $\mathbf{N}^{\mathbf{a}}$ & CCA $(\%)$ & No CCA (\%) & OR $(95 \%$ CI $)$ \\
\hline Overall & $47,446,658$ & & & $\mathrm{n} / \mathrm{a}$ \\
\hline \multicolumn{5}{|l|}{ Maternal age (years) } \\
\hline$<20$ & $5,179,407$ & 6.43 & 10.92 & $0.50(0.40-0.61)$ \\
\hline $20-24$ & $11,694,618$ & 27.05 & 26.64 & $0.93(0.81-1.06)$ \\
\hline $25-29$ & $12,899,876$ & 32.21 & 27.19 & Reference \\
\hline $30-34$ & $11,020,945$ & 20.74 & 23.23 & $0.75(0.65-0.88)$ \\
\hline$\geq 35$ & $6,626,859$ & 13.57 & 13.97 & $0.82(0.70-0.96)$ \\
\hline \multicolumn{5}{|l|}{ Maternal race } \\
\hline White-NH & $18,951,456$ & 45.37 & 39.94 & Reference \\
\hline Black-NH & $4,670,322$ & 9.53 & 9.84 & $0.85(0.70-1.04)$ \\
\hline Hispanic & $8,203,578$ & 15.60 & 17.29 & $0.79(0.63-1.00)$ \\
\hline Other-NH & $3,599,691$ & 3.74 & 7.59 & $0.43(0.33-0.57)$ \\
\hline Missing/Unknown & $12,021,611$ & 25.76 & 25.33 & $0.90(0.76-1.05)$ \\
\hline Tobacco use $e^{\mathrm{b}}$ & $1,610,608$ & 13.29 & 3.39 & $4.36(3.74-5.09)$ \\
\hline Alcohol use ${ }^{\mathrm{b}}$ & 46,113 & 0.53 & 0.10 & $5.51(2.44-12.46)$ \\
\hline Drug use ${ }^{\mathrm{b}}$ & 486,005 & 2.72 & 1.02 & $2.71(1.93-3.81)$ \\
\hline \multicolumn{5}{|l|}{ Hospital region } \\
\hline Northeast & $7,937,070$ & 13.30 & 16.73 & $0.96(0.67-1.37)$ \\
\hline Midwest & $10,249,569$ & 24.86 & 21.60 & $1.39(1.13-1.72)$ \\
\hline South & $17,498,148$ & 41.34 & 36.88 & $1.36(1.10-1.68)$ \\
\hline West & $11,761,871$ & 20.50 & 24.79 & Reference \\
\hline \multicolumn{5}{|l|}{ Hospital location } \\
\hline Rural & $5,893,039$ & 15.87 & 12.45 & Reference \\
\hline Urban & $41,432,694$ & 84.13 & 87.55 & $0.75(0.63-0.91)$ \\
\hline \multicolumn{5}{|l|}{ Hospital teaching status } \\
\hline Non-teaching & $25,549,873$ & 47.24 & 53.99 & Reference \\
\hline Teaching & $21,775,860$ & 52.76 & 46.01 & $1.31(1.12-1.54)$ \\
\hline \multicolumn{5}{|l|}{ Hospital Bed size } \\
\hline Small & $5,324,708$ & 10.80 & 11.25 & $1.00(0.80-1.24)$ \\
\hline Medium & $12,660,955$ & 25.77 & 26.75 & Reference \\
\hline Large & $29,340,070$ & 63.43 & 62.00 & $1.06(0.89-1.27)$ \\
\hline \multicolumn{5}{|l|}{ Household income } \\
\hline Lowest quartile & $12,143,344$ & 34.21 & 25.59 & $2.30(1.92-2.75)$ \\
\hline $2^{\text {nd }}$ quartile & $11,981,361$ & 28.58 & 25.25 & $1.95(1.64-2.32)$ \\
\hline $3^{\text {rd }}$ quartile & $11,419,328$ & 21.61 & 24.07 & $1.54(1.30-1.84)$ \\
\hline Highest quartile & $11,092,423$ & 13.59 & 23.38 & Reference \\
\hline Missing/Unknown & 810,202 & 2.01 & 1.71 & $2.03(1.38-2.97)$ \\
\hline \multicolumn{5}{|l|}{ Primary payer } \\
\hline Medicare/Medicaid & $18,723,901$ & 1.61 & 39.46 & $1.82(1.60-2.07)$ \\
\hline Private & $25,662,632$ & 1.67 & 54.09 & Reference \\
\hline Other & $3,060,125$ & 0.73 & 6.45 & $1.38(1.09-1.75)$ \\
\hline Clinical comorbidities & $1,963,585$ & 5.87 & 4.14 & $1.45(1.19-1.76)$ \\
\hline Pregnancy comorbidities & $5,663,671$ & 13.91 & 11.94 & $1.19(1.04-1.37)$ \\
\hline$S T D$ & 60,740 & 0.34 & 0.13 & $2.67(1.67-6.09)$ \\
\hline$H I V$ & 51,071 & 0.66 & 0.11 & $6.19(3.55-10.79)$ \\
\hline Obesity & 626,608 & 1.50 & 1.32 & $1.14(0.77-1.67)$ \\
\hline
\end{tabular}

$\mathbf{C C A}=$ cervical cancer, $\mathbf{N H}=$ non-Hispanic, $\mathbf{O R}=$ odds ratio, $\mathbf{C I}=$ confidence interval, $\mathbf{S T D}=$ sexually transmitted disease, $\mathbf{H I V}=$ human immunodeficiency virus, ${ }^{\mathrm{a}} \mathrm{Weighted}$ to estimate national frequency; sum of all groups may not add up to the total due to missing data, ${ }^{\mathrm{b}}$ Reference group is represented by the absence of the condition/characteristic. 


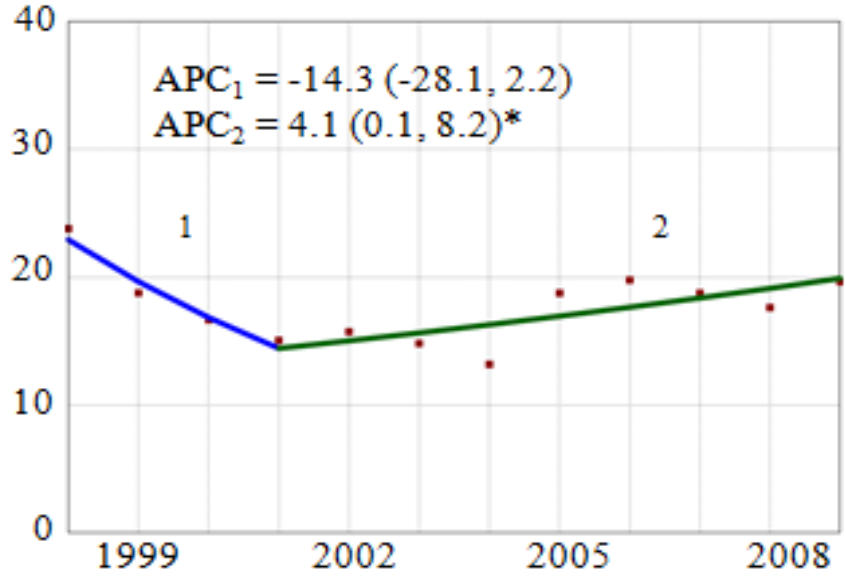

Fig. (1). Overall Trend and Annual Percent Change (APC) of Cervical Cancer Diagnosis Among Delivery-Related Discharges in the United States by Region, HCUP-NIS, 1998-2009. APC = annual percent change, point estimate ( $95 \%$ confidence interval). Vertical axis: diagnosed Cervical Cancer per 100,000 discharges; Horizontal axis: year of study. *The APC for the joinpoint segment is statistically significantly different from zero $(\mathrm{p}<0.05)$.

Age-specific trend analyses showed a statistically significant 10.6\% (95\% CI: 4.6, 16.9) annual increase in pregnancy-related cervical cancer since 2001 among women aged 20-24 years (Fig. 2). Conversely, among women 25-29 years old, there was an average $1.9 \%$ (95\% CI: $-4.8,-1.2)$ decrease in the rate during the study period. For other age groups, the rate of pregnancy-related cervical cancer was either flat over time (30-34 years), or erratic without a statistically significant trend $(<20,30-34$, and $35+$ years $)$. We observed tendencies towards racial/ethnic differences in the both the rates and trends of pregnancy-related cervical cancer. There was an estimated $1.3 \%$ and $2.0 \%$ annual reduction for white-NH and black-NH, respectively (data not shown), and annual increases for Hispanics ( $\mathrm{APC}=2.7 \%$ ), and other-NH $(\mathrm{APC}=6.0 \%)$. Despite the differences in their point estimates, none of these trends were statistically significantly different from zero.

Table 2 presents the associations between pregnancyrelated cervical cancer and selected maternal-fetal outcomes. After controlling for potential sociodemographic, behavioral, clinical, and hospital-related confounders, we observed between a 51\% and 95\% increased odds of adverse pregnancy outcomes among women with a cervical cancer diagnosis at the time of their delivery hospitalization. Women with a diagnosis of cervical cancer had 95\% higher odds of being diagnosed with anxiety $(95 \% \mathrm{CI}=1.11-3.42)$; $78 \%$ higher odds of anemia $(95 \% \mathrm{CI}=1.54-2.06)$; and $69 \%$ higher odds of preterm labor $(95 \% \mathrm{CI}=1.46-1.97)$ than women without cervical cancer. We also observed an increased odds of delivery by cesarean section $(\mathrm{AOR}=1.67$, $95 \% \mathrm{CI}=1.46-1.90)$ and a prolonged hospital stay $(\mathrm{AOR}=$ $1.51,95 \% \mathrm{CI}=1.30-1.76)$ among women pregnancy-related cervical cancer. These adverse maternal-fetal outcomes translated into differences in the mean, per hospitalization, cost of inpatient medical care between women with $(\$ 4,745)$
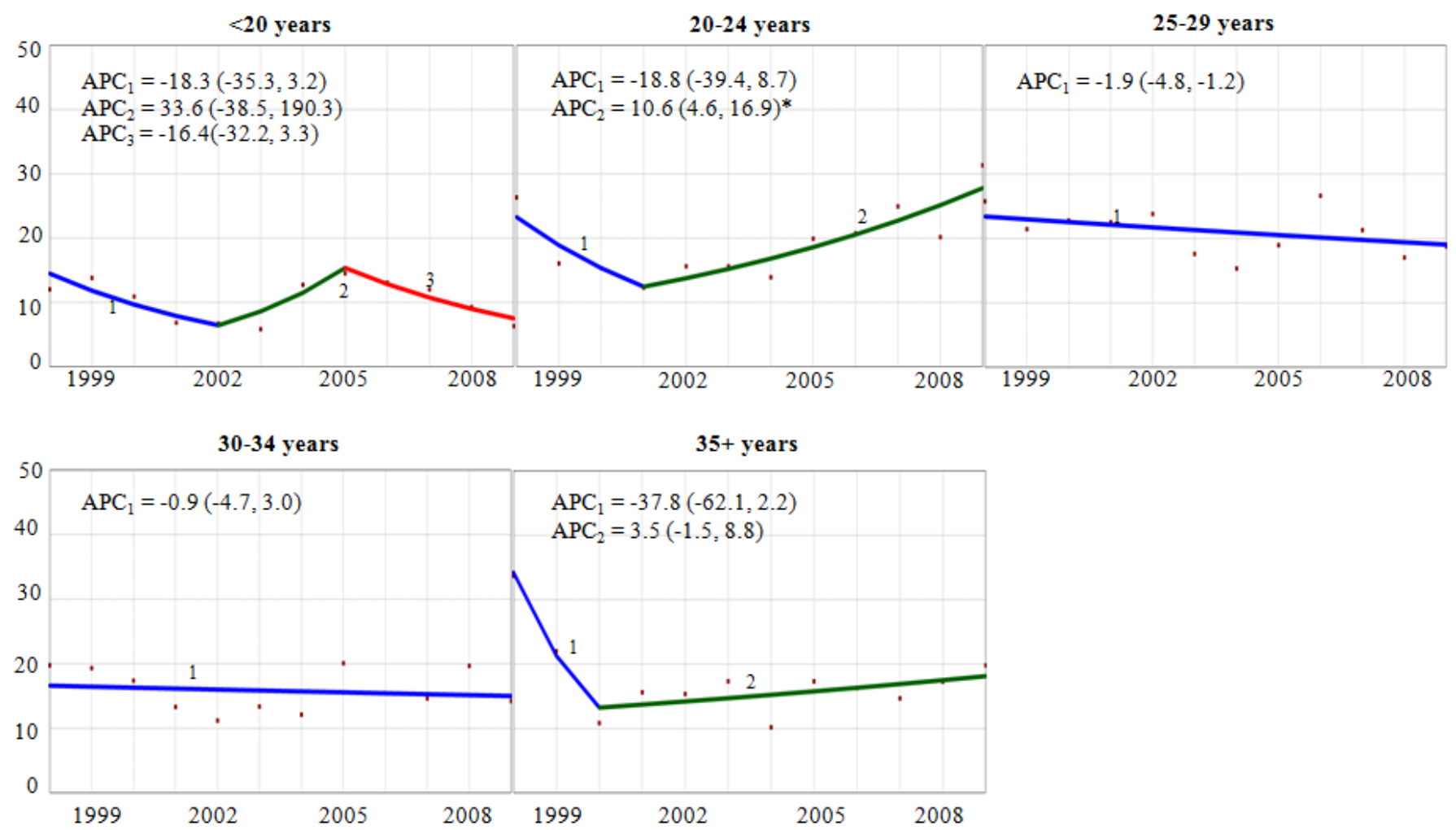

Fig. (2). Trend and Annual Percent Change (APC) of Cervical Cancer Diagnosis Among Delivery-Related Discharges in the United States by Maternal Age Group, HCUP-NIS, 1998-2009. APC = annual percent change, point estimate (95\% confidence interval). Vertical axis: diagnosed cervical cancer per 100,000 discharges. Horizontal axis: year of discharge. *The APC for the joinpoint segment is statistically significantly different from zero $(\mathrm{p}<0.05)$. 
with without $(\$ 3,986)$ pregnancy-related cervical cancer $(p<0.01)$, even when restricted to consideration of only the delivery hospitalization.

\section{DISCUSSION}

Albeit a relatively rare occurrence, cervical cancer during pregnancy is a tragic event that can pose life-threatening risks for the mother and developing fetus. Since more than $98.5 \%$ of all births in the US occur in a healthcare setting [18], analyzing a nationally representative sample of inpatient hospitalizations could provide an accurate and reliable estimate of the prevalence of pregnancy-related cervical cancer. To our knowledge, this is the largest study in the US to assess the temporal trends in cervical cancer diagnoses among delivery hospitalizations and to investigate simultaneously the associations between cervical cancer and maternal-fetal morbidities. Previous studies on cervical cancer focused on trends among all women $[4,19,20]$ and those that studied the prevalence within a pregnant population failed to assess its association with maternal comorbidities or pregnancy outcomes [5, 21-23]. The findings of this study contribute significantly to the literature by providing a basis for establishing recent rates and trends of pregnancy-related cervical cancer, assessing the increased risk for adverse outcomes that may be imposed by cervical cancer during pregnancy, and how that translates into differences in the direct medical costs associated with inpatient care at delivery.

In our 12-year national study, we observed a steady increase in the pregnancy-related cervical cancer from 20012009. This is consistent a large study in Denmark that reported a trend of increasing prevalence of cervical cancer among pregnant mothers over the past 30 years (crude $\mathrm{APC}=0.8 \%$; $95 \%$ CI: -0.6 to 2.1 ) [5]. Considering the near universal availability of screening and vaccination programs in the US, the increasing trend in pregnancy-related cervical cancer is of concern. One possible explanation for our findings may be a product of the limitation of the NIS data the unit of analysis is a single discharge record, and discharges for the same woman cannot be linked together. Therefore, there is a potential for counting a diagnosis of cervical cancer for a woman multiple times. To increase the likelihood that a cervical cancer diagnosis for a particular woman (or for a particular pregnancy) is only counted once, we restricted our analyses to delivery hospitalizations. By broadening our scope to include all pregnancy-related discharge records, we would probably improve slightly our ability to capture cases of cervical cancer, but would very likely overestimate the prevalence of pregnancy-related cervical cancer due to the aforementioned consequence of multiple counting. Another plausible explanation for the increasing trend is that because of an increased frequency of medical encounters during pregnancy (e.g. antenatal care visits) women are more likely to have better awareness and consent to screening $[4,6]$. One may also speculate that the increasing trend could be because of the concomitant increase in human papillomavirus (HPV) infections. Since our data do not provide information on HPV infection, we analyzed the prevalence and trends of a broader group of STI infections $[24,25]$. As hypothesized, we observed an $11.2 \%$ $[95 \% \mathrm{CI}=9.2-13.3]$ annual increase in the prevalence of STIs from 2000-2009, which could, in part, explain the $4.1 \%$ average annual increase in the pregnancy-related cervical cancer rate from 2001-2009.

In line with recent studies of cervical cancer in the general population $[4,26,27]$, stratified analysis by race/ethnicity revealed a bigger decrease in pregnancyrelated cervical cancer among black-NH women than among white-NH women. Unlike black-NH and white-NH women, cervical cancer rates for Hispanic women increased, on

Table 2. Outcome rates, adjusted odds ratios, and $95 \%$ confidence intervals for the association between CCA and maternal-infant morbidity and mortality, HCUP-NIS, 1998-2009.

\begin{tabular}{|c|c|c|c|c|c|}
\hline \multirow{2}{*}{ Outcomes } & \multicolumn{2}{|c|}{ Rate $^{a}$} & \multicolumn{3}{|c|}{ OR $(95 \%$ CI $)$} \\
\hline & CCA & No CCA & Model $1^{\mathrm{b}}$ & Model 2 & Model 3 $^{\mathrm{d}}$ \\
\hline \multicolumn{6}{|c|}{ Maternal, Pregnancy-related } \\
\hline Cesarean section $^{\mathrm{e}}$ & 346.2 & 274.7 & $1.40(1.25-1.57)$ & $1.69(1.49-1.92)$ & $1.67(1.46-1.90)$ \\
\hline Hospital stay $\geq 4$ days $^{\mathrm{f}}$ & 167.6 & 122.9 & $1.44(1.24-1.66)$ & $1.54(1.33-1.77)$ & $1.51(1.30-1.76)$ \\
\hline \multicolumn{6}{|c|}{ Maternal, Clinical Conditions } \\
\hline Anemia & 142.8 & 80.0 & $1.92(1.67-2.20)$ & $1.80(1.56-2.07)$ & $1.78(1.54-2.06)$ \\
\hline Anxiety & 9.3 & 3.4 & $2.74(1.58-4.78)$ & $2.02(1.16-3.53)$ & $1.95(1.11-3.42)$ \\
\hline \multicolumn{6}{|l|}{ Infant outcomes } \\
\hline Poor fetal growth & 24.7 & 16.0 & $1.57(1.16-2.12)$ & $1.35(1.01-1.82)$ & $1.32(0.98-1.78)$ \\
\hline Preterm delivery & 114.7 & 65.5 & $1.85(1.60-2.14)$ & $1.73(1.49-2.00)$ & $1.69(1.46-1.97)$ \\
\hline
\end{tabular}

$\boldsymbol{C C A}=$ cervical cancer, $\boldsymbol{O} \boldsymbol{R}=$ odds ratio, $\boldsymbol{C I}=$ confidence interval

${ }^{\text {a }}$ Per 1,000 pregnancy-related discharges.

'Unadjusted model with the presence of the condition as the outcome, $C C A$ status as the exposure ("No $C C A$ " is the reference group).

${ }^{\mathrm{c}}$ Model 1 + adjustment for maternal age, race/ethnicity, household income, tobacco, alcohol, and drug use, primary payer, rural/urban status, and obesity

${ }^{\mathrm{d}}$ Model 2 + additional adjustment for composite of clinical (coronary heart disease, hyperlipidemia, hypothyroidism, disorders of the adrenal gland, existing hypertension, diabetes mellitus, and depression) and pregnancy (preeclampsia, eclampsia, placenta abruptio, placenta accreta, placenta previa, gestational diabetes, and gestational hypertension) related conditions of the mother.

${ }^{\mathrm{e}}$ Additional adjustment for previous Cesarean section in Models 2 and 3.

${ }^{\mathrm{f}}$ Additional adjustment for discharge disposition status in Models 2 and 3. 
average, $2.7 \%$ annually. Part of the racial/ethnic disparity could be due to variation in the utilization of cervical cancer prevention programs $[28,29]$. A study based on analysis of data from the National Immunization Survey among teenage girls (13-17 years) reported lower rates of completion of the required HPV vaccine regimen among blacks and Hispanics $[30,31]$. However, the racial/ethnic disparities in pregnancyrelated cervical cancer warrants further investigation.

Differences in pregnancy-related cervical cancer rates by geographic region of the US could be explained by baseline differences in the distribution of socio-demographic makeup of their respective populations, as well as in the prevalence of clinical and pregnancy-related complications. In assessing geographic differences, we attempted to adjust for patient and hospital characteristics; however, some residual confounding may persist and explain some of those findings. Stratified trend analyses by age demonstrated $10.6 \%$ and $3.5 \%$ annual increases for women $20-25$ years and $\geq 35$ years of age respectively. The former peak (20-25 years) could be related to women becoming sexually active in their early teens [32] increasing the risk of being infected with HPV, a critical factor for cervical cancer [33]. The increasing rates observed among women of advanced maternal age (age $\geq 35$ years) could be due to the increased risk associated with having a first pregnancy later in life [34]. Unfortunately, neither parity nor time at first delivery is available in the NIS; therefore, we could not further investigate this hypothesis.

The increased rate of cesarean section and preterm delivery observed in our study are consistent with the results of previous studies that have investigated the impact of pregnancy-related reproductive or non-reproductive cancers $[23,35]$. The increased likelihood of these adverse outcomes among woman with cervical cancer in pregnancy are in line with the theory that clinically aggressive types of cancers are more likely to be diagnosed at the time of delivery than less aggressive cancers. Diagnosis early in pregnancy could lead to a decision to deliver earlier by cesarean section, so that treatment of the aggressive cancer can begin as early as possible. Future studies with more in-depth clinical information should investigate the association between cervical cancer and pregnancy outcomes, stratified by histologic subtype.

Women with cervical cancer during pregnancy were $78 \%$ more likely to experience anxiety. A previous study reported symptoms of anxiety in patients receiving cervical cancer treatment [36] and long term cancer survivors [37]. The increased level of anxiety for a woman diagnosed with cervical cancer is likely due to increased concern for existential well-being of herself and her baby [38, 39]. Anemia is one of the major complications of many cancers [40], and impacts more than $40 \%$ of cancer patients [41]. It is established that women who are anemic during pregnancy are more likely to give birth to a preterm infant $[42,43]$. In our study, women with cervical cancer were $95 \%$ more likely to be diagnosed with anemia. Therefore, the observed increased risk of preterm birth among infants born to women with cervical cancer could be explained partly by the higher prevalence of anemia among cervical cancer patients [35, 44].

The results of this study should be interpreted with the following limitations in mind. First, because of the administrative nature of the NIS data, we relied on ICD-9$\mathrm{CM}$ codes to identify cases of cervical cancer, clinical and pregnancy-related comorbidities, and behavioral conditions. These data are subject to errors in coding, which could lead to over- or under estimation of cases and other comorbidities. Second, it is possible that women with a cervical cancer diagnosis during pregnancy could receive more thorough assessment, follow-up, and extensive documentation of behavioral and clinical comorbidities compared to other pregnant women, which could lead to a diagnostic bias that results in differential misclassification and an overestimation of the measures of associations we report. Third, some states do not report race/ethnicity data $(25 \%$ of discharges in this study were missing race/ethnicity), and the states that do categorize race/ethnicity do so in a non-uniform manner. HCUP attempts to standardize categorization of race/ethnicity to enabling comparisons but one should still interpret those findings with caution. Despite these known limitations of the NIS dataset, the large and representative sample of inpatient hospital discharges affords an efficient means of generating national estimates of pregnancy-related cervical cancer rates and trends. The substantial sample size also permitted our investigation of trends within geographic region and sociodemographic subgroups.

In conclusion, there is a recent increase in the prevalence of cervical cancer during pregnancy. There is also evidence that pregnancy-related cervical cancer increases the likelihood of several maternal co-morbidities and adverse pregnancy outcomes. Interventions that promote safer sexual practice and regular screening for cervical cancer should be promoted widely among women of reproductive age in order to effectively reduce the prevalence of cervical cancer during pregnancy and its impact on the health of mother and baby.

\section{CONFLICT OF INTEREST}

The authors confirm that this article content has no conflict of interest.

\section{ACKNOWLEDGEMENTS}

This work partly supported by: (1) the National Institutes of Health through a grant on Cancer health disparities (award no. 1P20MD003375-01) and the Agency for Health Care Research, (2) Quality through a grant on Clinically Enhanced MultiPurpose Administrative Data set for Comparative Effectiveness Research (award no. 1R0111HS019997) and (3) Indiana University School of Nursing. 


\section{APPENDIX A}

\section{List of International Classification of Diseases, $9^{\text {th }}$ Edition, Clinical Modification Codes Used to Identify Selected Clinical and Behavioral Conditions.}

\begin{tabular}{|c|c|}
\hline Condition & International Classification of Diseases, $9^{\text {th }}$ Edition, Diagnosis Code ${ }^{\mathrm{a}}$ \\
\hline \multicolumn{2}{|l|}{ Exposure } \\
\hline Cervical cancer & $180,795.06,233.1$ \\
\hline \multicolumn{2}{|l|}{ Comorbidities } \\
\hline Obesity & $278.00,278.01,278.03,649.1 x, V 85.3 x, V 85.4 x, V 85.54,793.91$ \\
\hline Pre-pregnancy hypertension & $401 x, 402 x, 403 x, 404 x, 405 x, 642.0 x, 642.1 x, 642.2 x, 642.7 x$ \\
\hline Pre-pregnancy diabetes & $249 x, 250 x, 648.0 x$ \\
\hline Coronary heart disease & $410 x, 411 x, 412 x, 413 x, 414 x, 429.2$ \\
\hline Chronic renal disease & $581 \mathrm{x}, 582 \mathrm{x}, 583 \mathrm{x}, 585 \mathrm{x}, 587 \mathrm{x}, 646.2 \mathrm{x}$ \\
\hline Anemia & $280 x, 281 x, 282 x, 283 x, 284 x, 285 x, 648.2 x$ \\
\hline Disorders of lipid metabolism (e.g., hyperlipidemia) & $272 \mathrm{x}$ \\
\hline Hypothyroidism & $243 x, 244 x$ \\
\hline Disorders of the adrenal glands & $255 \mathrm{x}$ \\
\hline Cesarean section ${ }^{\mathrm{b}}$ & $74 \mathrm{x}$ \\
\hline Gestational diabetes & $648.8 \mathrm{x}$ \\
\hline Gestational hypertension & $642.3 x$ \\
\hline Pre-eclampsia & $642.4 x, 642.5 x$ \\
\hline Eclampsia & $642.6 x$ \\
\hline Acute renal failure & $584 x, 669.3 x$ \\
\hline HIV & 042x, 043x, 044x, 079.53, 279.10, 279.19, 795.71, 795.8, V08 \\
\hline STD & $\begin{array}{c}\text { 090.x, 091.x, 092.x, 093.x, 094.x, 095.x, 096.x, 097.x, 098.x, 099.x, 795.05, 795.15, 795.19, } \\
\text { 796.75, 796.69 }\end{array}$ \\
\hline \multicolumn{2}{|l|}{ Perinatal history } \\
\hline Previous Cesarean section & $654.2 \mathrm{x}$ \\
\hline \multicolumn{2}{|l|}{ Behavioral history } \\
\hline Tobacco use & $305.1,649.0 x, 989.84$ \\
\hline Alcohol use & $291 x, 303 x, 305.0 x, 425.5,760.71, \mathrm{~V} 11.3$ \\
\hline Drug use & $\begin{array}{c}292.0 \mathrm{x}, 292.1 \mathrm{x}, 292.2 \mathrm{x}, 292.8 \mathrm{x}, 304 \mathrm{x}, 305.2 \mathrm{x}, 305.3 \mathrm{x}, 305.4 \mathrm{x}, 305.5 \mathrm{x}, 305.6 \mathrm{x}, 305.7 \mathrm{x}, 305.9 \mathrm{x}, \\
648.3 \mathrm{x}, 655.5 \mathrm{x}, 760.72,779.5,760.75,965.00,965.02, \mathrm{E} 935.1, \mathrm{E} 850.1\end{array}$ \\
\hline \multicolumn{2}{|l|}{ Maternal outcomes } \\
\hline Anemia & $280 x, 281 x, 282 x, 283 x, 284 x, 285 x, 648.2 x$ \\
\hline \multicolumn{2}{|l|}{ Fetal outcomes } \\
\hline Preterm labor & $644.2 x$ \\
\hline Poor fetal growth & $656.5 x$ \\
\hline
\end{tabular}

${ }^{a}$ The code suffix " $x$ " represents all possible codes that follow the stated code prefix. ${ }^{b}$ procedure codes, not diagnostic codes were used to define Cesarean section

\section{REFERENCES}

[1] Matthews TJ, Hamilton BE. Delayed childbearing: more women are having their first child later in life. NCHS Data Brief 2009 21:1-8.

[2] Peccatori FA, Azim HA, R, Hoekstra HJ, et al. Cancer, pregnancy and fertility: ESMO Clinical Practice Guidelines for diagnosis, treatment and follow-up. Ann Oncol 2013; 24 (6): 60-70.
[3] Stensheim H, Moller B, van Dijk T, Fossa SD. Cause-specific survival for women diagnosed with cancer during pregnancy or lactation: a registry-based cohort study. J Clin Oncol 2009; 27(1): 45-51.

[4] Adegoke O, Kulasingam S, Virnig B. Cervical cancer trends in the United States: a 35-year population-based analysis. J Women's Health 2002; 21(10): 1031-7. 
[5] Eibye S, Kjaer SK, Mellemkjaer L. Incidence of pregnancyassociated cancer in Denmark, 1977-2006. Obstet Gynecol 2013;122(3): 608-17.

[6] Hui SK, Engelman KK, Shireman TI, Ellerbeck EF. Adherence to cancer screening guidelines and predictors of improvement among participants in the Kansas State Employee Wellness Program. Preve Chron Dis 2013;10: E115.

[7] MacDorman MF, Mathews TJ, Declercq E. Home births in the United States, 1990-2009. NCHS Data Brief 2012; 84:1-8.

[8] Introduction to the HCUP nationwide inpatient sample (NIS) Rockville, MD: Agency for Healthcare Research and Quality; 2011 Available from: [http://www.hcup-us.ahrq.gov/db/nation/nis/NIS_I ntroduction_2011.jsp]

[9] Merrill C, Owens PL. Reasons for Being Admitted to the Hospital through the Emergency Department for Children and Adolescents, 2004: Statistical Brief \#33. 2006. In: Healthcare Cost and Utilization Project (HCUP) Statistical Briefs [Internet]. Rockville MD.

[10] HCUP quality control procedures 2008 [cited 2015 March 20]. Available from:[http://www.hcup-us.ahrq.gov/db/quality.pdf]

[11] Finkler SA. The distinction between cost and charges. Ann Intern Med 1982; 96(1):102-9.

[12] Salemi JL, Comins MM, Chandler K, Mogos MF, Salihu HM. A practical approach for calculating reliable cost estimates from observational data: application to cost analyses in maternal and child health. App Health Econ Health Policy 2013; 11(4): 343-57.

[13] Cost-to-Charge Ration Files (CCR). Healthcare Cost and Utilization Project (HCUP) 2001-2009 Rockville, MD2011 [May 9, 2013]. Available from: http://www.hcup-us.ahrq.gov/db/state/co sttocharge.jsp.

[14] Sun Y, Friedman B. Tools for more accurate inpatient cost estimates with HCUP databases, 2009. Errata added October 25, 2012. HCUP Methods Series Report \# 2011-04. 2012 [cited 2013 May 14]. Available from: http://www.hcup-us.ahrq.gov/reports/me thods/2011_04.pdf.

[15] Kim HJ, Fay MP, Feuer EJ, Midthune DN. Permutation tests for joinpoint regression with applications to cancer rates. Stat Med 2000; 19(3): 335-51.

[16] Houchens RL, Elixhauser A. Using the HCUP nationwide inpatient sample to estimate trends (updated for 1988-2004). HCUP methods series Report \# 2006-05 online. 2006. Available from: [http://www.hcupus.ahrq.gov/reports/methods/2006_05_NISTrends Report 1988-2004.pdf]

[17] Joinpoint Regression Program, Version 4.0.1 - January 2013; Statistical Methodology and Applications Branch and Data Modeling Branch, Surveillance Research Program National Cancer Institute 2013.

[18] MacDorman MF, Matthews TJ, Declercq E. Trends in out-ofhospital births in the United States, 1990-2012. NCHS Data Brief. 2014; (144):1-8.

[19] Saraiya M, Ahmed F, Krishnan S, Richards TB, Unger ER, Lawson HW. Cervical cancer incidence in a prevaccine era in the United States, 1998-2002. Obstet Gynecol 2007; 109: 360-70.

[20] Sherman ME, Wang SS, Carreon J, Devesa SS. Mortality trends for cervical squamous and adenocarcinoma in the United States. Relation to incidence and survival. Cancer 2005; 103(6):1258-64.

[21] Pettersson BF, Andersson S, Hellman K, Hellstrom AC. Invasive carcinoma of the uterine cervix associated with pregnancy: 90 years of experience. Cancer 2010; 116(10): 2343-9.

[22] Smith LH, Dalrymple JL, Leiserowitz GS, Danielsen B, Gilbert WM. Obstetrical deliveries associated with maternal malignancy in California, 1992 through 1997. Am J Obstet Gynecol 2001; 184(7): 1504-12.

[23] Smith LH, Danielsen B, Allen ME, Cress R. Cancer associated with obstetric delivery: results of linkage with the California cancer registry. Am J Obstet Gynecol 2003; 189(4): 1128-35.
[24] Burchell AN, Winer RL, de Sanjose S, Franco EL. Epidemiology and transmission dynamics of genital HPV infection. Vaccine 2006; 24(3): 52-61.

[25] Winer RL, Lee SK, Hughes JP, Adam DE, Kiviat NB, Koutsky LA. Genital human papillomavirus infection: incidence and risk factors in a cohort of female university students. Am J Epidemiol 2003; 157(3): 218-26.

[26] Cervical Cancer 2013 [updated December 9, 2013; cited 2015 March 20]. Available from: [http://www.cancer.org/acs/groups/cid/ documents/webcontent/003094-pdf.pdf]

[27] Doescher MP, Jackson JE. Trends in cervical and breast cancer screening practices among women in rural and urban areas of the United States. J Public Health Manag Pract 2009; 15(3): 200-9.

[28] Widdice LE, Bernstein DI, Leonard AC, Marsolo KA, Kahn JA. Adherence to the HPV vaccine dosing intervals and factors associated with completion of 3 doses. Pediatrics 2011; 127(1): 7784.

[29] McDougall JA, Madeleine MM, Daling JR, Li CI. Racial and ethnic disparities in cervical cancer incidence rates in the United States, 1992-2003. Cancer Causes |Control CCC 2007; 18(10): 1175-86.

[30] Niccolai LM, Mehta NR, Hadler JL. Racial/Ethnic and Poverty Disparities in Human Papillomavirus Vaccination Completion. Am J Prev Med 2011; 41(4): 428-33.

[31] Elam-Evans LD, Yankey D, Jeyarajah J, et al. National, regional, state, and selected local area vaccination coverage among adolescents aged 13-17 years--United States, 2013. Morb Mortal Wkly Rep 2014; 63(29): 625-33.

[32] Foley G, Alston R, Geraci M, Brabin L, Kitchener H, Birch J. Increasing rates of cervical cancer in young women in England: an analysis of national data 1982-2006. Br J Cancer 2011; 105(1): 177-84.

[33] Bosch FX, Lorincz A, Munoz N, Meijer CJ, Shah KV. The causal relation between human papillomavirus and cervical cancer. J Clin Pathol 2002; 55(4): 244-65.

[34] Ventura SJ, Curtin SC, Abma JC, Henshaw SK. Estimated pregnancy rates and rates of pregnancy outcomes for the United States, 1990-2008. National Center Health Stat 2012; 60(7): 1-21.

[35] Mogos MF, Salihu HM, Aliyu MH, Whiteman VE, Sultan DH. Association between reproductive cancer and fetal outcomes: a population-based study. Int J Gynecol Cancer 2013; 23(2): 218-26.

[36] Conic I, Miljkovic S, Tosic-Golubovic S, et al. Anxiety levels related to the type of therapy for cervical cancer. Centeur J Med 2012; 7(4): 490-6.

[37] Mitchell AJ, Ferguson DW, Gill J, Paul J, Symonds P. Depression and anxiety in long-term cancer survivors compared with spouses and healthy controls: a systematic review and meta-analysis. Lancet Oncol 2013;14(8): 721-32.

[38] Salz T, Gottlieb SL, Smith JS, Brewer NT. The association between cervical abnormalities and attitudes toward cervical cancer prevention. J Women's Health 2010; 19(11): 2011-6.

[39] Kim SH, Kang S, Kim YM, et al. Prevalence and predictors of anxiety and depression among cervical cancer survivors in Korea. Int J Gynecol 2010; 20(6):1017-24.

[40] Candelaria M, Cetina L, Duenas-Gonzalez A. Anemia in cervical cancer patients: implications for iron supplementation therapy. Med Oncol 2005; 22(2): 161-8.

[41] Knight K, Wade S, Balducci L. Prevalence and outcomes of anemia in cancer: a systematic review of the literature. Am J Med 2004;116 (7): 11S-26S.

[42] Levy A, Fraser D, Katz M, Mazor M, Sheiner E. Maternal anemia during pregnancy is an independent risk factor for low birthweight and preterm delivery. Eur J Obst Gynecol 2005; 122(2): 182-6.

[43] Zhang Q, Ananth CV, Li Z, Smulian JC. Maternal anaemia and preterm birth: a prospective cohort study. Int J Epidemiol 2009; 38(5): 1380-9.

[44] Weisz B, Schiff E, Lishner M. Cancer in pregnancy: maternal and fetal implications. Hum Reprod 2001; 7(4): 384-93. 